'Unidad de Neurología Cognitiva y Demencias, Servicio de Neurología, Hospital del Salvador. Santiago, Región Metropolitana, Chile. Universidad de Chile.

${ }^{2}$ Departamento de

Psicología, Facultad de Ciencias Sociales, Universidad de Chile.

${ }^{3}$ Centro de Investigación Avanzada en Educación, Universidad de Chile.

${ }^{4}$ Departamento de Farmacología Molecular y Clínica, ICBM y Departamento de Ciencias Neurológicas Oriente, Facultad de Medicina, Universidad de Chile, Santiago, Chile

${ }^{5}$ Servicio de Neurología,

Clínica Alemana de Santiago, Región Metropolitana, Chile. assicólogo(a)

Financiamiento FONDECYT Proyecto No 1100975 PIA-Conicyt Proyecto CIE-05

Los autores no tienen conflictos de intereses que declarar.

Recibido el 25 de agosto de 2011, aceptado el 4 de abril de 2012

Correspondencia a:

Ps. Carlos Muñoz Neira Unidad de Neurología Cognitiva y Demencias, Servicio de Neurología, Hospital del Salvador. Avenida Salvador 364, Santiago, Región Metropolitana, Chile. Teléfono: + 56-2-5754021

Fax: +56-2-5754021

Email:carlos7munoz@ gmail.com

\section{Propiedades psicométricas y utilidad diagnóstica del Addenbrooke's Cognitive Examination-Revised (ACE-R) en una muestra de ancianos chilenos}

\author{
CARLOS MUÑOZ-NEIRA ${ }^{1,2, a}$, FERNANDO HENRÍQUEZ CH. ${ }^{1,2, a}$, \\ JOSEFINA IHNEN J. ${ }^{1,2, a}$, MAURICIO SÁNCHEZ C. ${ }^{2, a}$, \\ PATRICIA FLORES M. ${ }^{1,3, a}$, ANDREA SLACHEVSKY CH. ${ }^{1,2,3,4,5}$
}

\section{Psychometric properties and diagnostic usefulness of the Addenbrooke's Cognitive Examination-revised in a Chilean elderly sample}

Background: The Addenbrooke's Cognitive Examination - Revised (ACE-R) is a good alternative to the Mini Mental State Examination (MMSE) for assessing cognitive capacities in dementia. Aim: To estimate the psychometric properties and diagnostic utility of the Addenbrooke's Cognitive Examination-Revised (ACE-R) in a Chilean elderly sample. Material and Methods: ACE-R was adapted for the Chilean population (ACE-R-Ch) and then administered to 60 dementia patients, 22 mild cognitive impairment (MCI) patients and 45 control subjects in addition to the MMSE for assessing global cognitive efficiency. Caregivers of dementia patients and collateral sources of MCI patients and elderly subjects without dementia were interviewed with measures of dementia severity, functional status in activities of daily living and cognitive changes. Convergent validity, internal consistency reliability, cutoff points, sensitivity and specificity for ACE-R-Ch were estimated. Results: Regarding convergent validity, the ACE- $R$-Ch showed significant correlations $(p<0,001)$ with another cognitive measure ( $r=0,952$ with MMSE), a rating for dementia severity (Spearman rho $=-0,822$ with CDR), functional capacity assessments $(r=-0,70$ with ADLQ-Ch; $r=-0,725$ with PFAQ-Ch; and $r=0,650$ with IADL Scale) and a measure of cognitive changes ( $r=-0,633$ with AD8-Ch). In terms of reliability, the test had a Cronbach alpha coefficient of 0.918. The best cut-off point to distinguish cases of dementia from control subjects was a score of 76 , which reached a sensitivity of 0.92 and a specificity of 0.93. Conclusions: The ACE-R-Ch showed acceptable psychometric properties, becoming a valid and reliable instrument to assess global cognitive efficiency or cognitive impairment. Its diagnostic utility to detect dementia patients also worked very well in a Chilean elderly sample.

(Rev Med Chile 2012; 140: 1006-1013).

Key words: Cognition; Dementia; Diagnostic techniques, neurological.

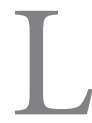

a utilidad de las pruebas de screening cognitivo con buenas propiedades psicométricas y capacidad diagnóstica para evaluar demencias es indiscutible. El test cognitivo breve más utilizado para ello es el Mini Mental State Examination (MMSE) $)^{1}$, ya estandarizado en Chile ${ }^{2}$. En términos de evaluaciones breves, con la intención de crear un test cognitivo simple para detectar precozmente las enfermedades demenciantes y ayudar a diferenciar entre sus distintos tipos, se desarrolló el Addenbrooke's Cognitive Examination $(\mathrm{ACE})^{3}$, instrumento que ha mostrado en diversos estudios mayor sensibilidad y valor predictivo para detectar demencias en comparación al MMSE, así 
como buena utilidad para discriminar entre enfermedad de Alzheimer, demencia frontotemporal, parálisis supranuclear progresiva y otros síndromes parkinsonianos que cursan con demencia ${ }^{3-7}$. Asimismo, ha sido sugerido que los síntomas anímicos comórbidos o trastornos del ánimo no afectarían su desempeño ${ }^{8}$.

Con el fin de facilitar su uso, proporcionar una herramienta que posibilite su traducción e implementación en otras culturas y mejorar su sensibilidad para detectar trastornos cognitivos, se diseñó la versión revisada del ACE: el Addenbrooke's Cognitive Examination-Revised (ACE-R $)^{9}$. El ACE-R incorpora en su estructura al MMSE, por lo que administrarlo conlleva la ventaja de obtener puntajes para ambas pruebas. Ha evidenciado una precisión diagnóstica y propiedades psicométricas similares a las del ACE, exhibiendo en su publicación original excelentes validez convergente, confiabilidad, sensibilidad y especificidad ${ }^{9}$. Considerando las características favorables de esta prueba y que los rendimientos en pruebas cognitivas son sensibles a variables socioculturales ${ }^{10}$, resulta relevante obtener datos chilenos para su utilización en la clínica de las demencias. Más aún, si se advierten las diversas limitaciones del MMSE ${ }^{11-15}$, se torna importante contar con una alternativa, en conjunto con disponer de los primeros datos de referencia acerca de la validez, confiabilidad, sensibilidad y especificidad para el ACE-R en Chile. El objetivo de este estudio entonces consistió en estimar las propiedades psicométricas y la utilidad diagnóstica del ACE-R en Chile (ACE-R-Ch) en una muestra de ancianos atendidos en el Servicio de Neurología del Hospital del Salvador incluyendo casos de demencia, deterioro cognitivo leve (DCL) y controles.

\section{Material y Método}

\section{Desarrollo del ACE-R-Ch}

El ACE-R original evalúa 5 dominios cognitivos: Orientación y Atención (18 puntos), Memoria (26 puntos), Fluencias Verbales (14 puntos), Lenguaje (26 puntos) y Habilidades Visoespaciales (16 puntos), siendo 100 puntos su puntaje máximo total. La adaptación y transculturación del ACE$\mathrm{R}$ se inició usando como referencia su versión en español (versión argentina) recientemente validada ${ }^{16}$. Se modificó la sub-escala de Memoria en la tarea de memoria anterógrada (prueba del
Nombre y la Dirección), además de las preguntas de memoria retrógrada. El ACE-R-Versión Chilena (ACE-R-Ch) (Anexo 1) alcanzó su versión definitiva después de un estudio piloto que incluyó a 30 sujetos normales. (El Anexo 1 no figura en esta publicación, pero puede solicitarse directamente al autor corresponsal).

\section{Pacientes}

Se realizó un muestreo por conveniencia, incluyendo a 127 adultos mayores de ambos sexos, 65 o más años de edad, habla hispana (chilenos) y con al menos 4 años de educación formal. Se consideraron 3 grupos para la configuración de la muestra: i) grupo de pacientes con demencia $(\mathrm{n}=60)$; ii) grupo de pacientes con DCL $(\mathrm{n}=22)$ y iii) grupo de control $(n=45)$. Se incorporaron en el estudio a pacientes de la Unidad de Neurología Cognitiva y Demencias del Servicio de Neurología del Hospital del Salvador. Los sujetos control correspondieron a cónyuges, familiares y personas cercanas a los consultantes de la unidad. Los diagnósticos fueron realizados por un neurólogo con la Clinical Dementia Rating Scale (CDR $)^{17,18}$ y los criterios diagnósticos establecidos para los diferentes tipos de demencia. Todos los sujetos incluidos en la investigación se sometieron a evaluaciones neurológica completa, neuropsicológica y con neuroimágenes (TAC o RMN) para confirmar su diagnóstico y ser correctamente categorizados. Contaban además con una fuente de garantía confiable (un cuidador primario para los pacientes con demencia y un informante clave para los pacientes con DCL y los controles). Se excluyeron del estudio a sujetos que presentaran déficits sensoriales severos (pérdida visión o audición) que imposibilitaran una evaluación neuropsicológica, depresión representados por un puntaje total $\geq 5$ puntos en la Geriatric Depression Scale ${ }^{19}$ y cuadros ansiosos determinados por un puntaje $\geq 51$ puntos en la Zung Scale ${ }^{20}$.

El grupo de pacientes con demencia incluyó a 60 sujetos de $C D R \geq 1^{17,18}$. Se incluyeron a 41 pacientes diagnosticados con enfermedad de Alzheimer según los criterios de la NINCDS$\mathrm{ADRDA}^{21}, 11$ con demencia frontotemporal según los criterios de Neary y Snowden ${ }^{22}, 5$ con demencia vascular según los criterios de la NINDS-AIREN ${ }^{23}$ y 3 con demencia por cuerpos de Lewy según los criterios de consenso para el cuadro ${ }^{24}$.

El grupo de pacientes con DCL incluyó a 22 
pacientes de $\mathrm{CDR}=0,5^{17,18}$. Se entendió por DCL un deterioro de uno o más dominios cognitivos con una capacidad funcional en las actividades de la vida diaria (AVD) relativamente preservada ${ }^{25}$.

El grupo control incluyó a 45 adultos mayores sanos de $C D R=0^{17,18}$ sin quejas de memoria ni historiales de enfermedades neurológicas y/o psiquiátricas.

\section{Evaluación y material utilizado}

Para obtener indicadores de eficiencia cognitiva global se le administró a toda la muestra el ACE-R-Ch en conjunto con el MMSE chileno ${ }^{2}$. La severidad de la demencia se estimó con la $\mathrm{CDR}^{17,18}$. La funcionalidad en las AVD se evaluó con el Activities of Daily Living Questionnaire en su versión validada en Chile (ADLQ-Ch) ${ }^{26}$, el Functional Assessment Questionaire (PFAQ) en su versión chilena ${ }^{27}$ y la Instrumental Activities of Daily Living (IADL) Scale ${ }^{28}$. Para medir la presencia de cambios cognitivos se usó la versión local del Alzheimer Disease 8 (AD8-Ch) ${ }^{29}$.

\section{Procedimiento y análisis de datos}

La investigación fue aprobada por el Comité de Ética Científico del Servicio de Salud Metropolitano Oriente. Todos los participantes firmaron un consentimiento informado previo a su inclusión al estudio. En algunos casos de demencia, el cuidador primario firmó el documento debido a la discapacidad cognitiva del paciente.

Luego del pilotaje, se realizó la recolección de datos y los análisis estadísticos respectivos con el Software Estadístico PASW Statistics 18 para Windows. Se obtuvieron los perfiles demográficos y clínicos de la muestra ordenados por división diagnóstica. Se compararon los 3 grupos estudiados (pacientes con demencia, DCL y controles) mediante pruebas ANOVA de un factor. Para estimar la validez convergente del instrumento ${ }^{30}$ se contrastaron mediante correlaciones los resultados obtenidos en el ACE-R-Ch con el MMSE ( $r$ de Pearson), la CDR (rho de Spearman), el ADLQ-Ch, el PFAQ, la IADL Scale y el AD8-Ch ( $\mathrm{r}$ de Pearson). Para determinar la confiabilidad del ACE-R-Ch se estimó su consistencia interna utilizando el coeficiente alfa de Cronbach ${ }^{31}$. Validez y confiabilidad se calcularon con todos los sujetos del estudio para utilizar una muestra de heterogeneidad máxima. Tanto los puntajes del ACE-R-Ch como los del MMSE se sometieron a un análisis con curvas ROC (Receiver Operator Characteristic) $^{32}$ para estimar los puntos de corte que permitieran entregar la sensibilidad y especificidad más altas diferenciando: i) pacientes con demencia de controles; ii) pacientes con DCL y demencia de controles, o bien iii) pacientes con DCL de controles. Para tales efectos, el diagnóstico clínico de demencia $(C D R \geq 1)$, así como el de DCL $(C D R=0,5)$ y el de un caso sano $(C D R=0)$, sirvieron como gold standard contra los cuales se compararon los puntajes del ACE-R-Ch y del MMSE. El área bajo la curva $(A B C)$ fue calculada también para cada situación de manera de precisar la utilidad diagnóstica de ambas pruebas. El nivel de significación estadística en todos los cálculos realizados fue $\leq 0,05$.

\section{Resultados}

\section{Adaptación y transculturización del ACE-R-Ch}

Un comité de jueces expertos ( 5 profesionales de la salud con al menos 5 años de experiencia en evaluaciones neuropsicológicas) consideró que el ACE-R-Ch se adaptó de forma apropiada, cumpliendo los criterios de pertinencia, claridad y suficiencia. Al ser probado en el estudio piloto, su aplicación no reveló dificultades. Durante la recolección de datos se observó que su administración es fácil y toma entre 20 y 40 minutos aproximadamente.

\section{Datos demográficos y perfiles clínicos}

En la Tabla 1 se resumen los datos demográficos y clínicos de la muestra separados en los 3 grupos establecidos: pacientes con demencia $(n=60)$, pacientes con DCL $(n=22)$ y sujetos control $(n=45)$. En términos de edad, años de educación y sexo no se encontraron diferencias significativas $(p>0,05)$ entre los grupos estudiados, situación que sustenta su homogeneidad. En cuanto a los perfiles clínicos, se encontraron diferencias significativas $(\mathrm{p}<0,01)$ y de efecto moderado $\left(0,47<\varepsilon^{2}<0,71\right)^{33}$ en medidas de eficiencia cognitiva global (ACER-Ch y MMSE), gravedad de la demencia (CDR), evaluación funcional (ADLQ-Ch, PFAQ e IADL Scale) y cuantificaciones de cambios cognitivos (AD8-Ch). El análisis post-hoc de estas variables identificó diferencias significativas $(p<0,05)$ y de amplio rango para todos los puntajes obtenidos en estas valoraciones entre los grupos de pacientes con demencia, DCL y controles, exceptuando dos 
Tabla 1. Datos demográficos y perfiles clínicos de la muestra

\begin{tabular}{|c|c|c|c|c|c|c|c|}
\hline \multirow[t]{2}{*}{ Parámetro } & \multicolumn{3}{|c|}{ Descriptivos según grupos } & \multicolumn{4}{|c|}{ Comparaciones por grupos } \\
\hline & $\begin{array}{c}\text { Demencia } \\
(n=60)\end{array}$ & $\begin{array}{c}\text { DCL } \\
(n=22)\end{array}$ & $\begin{array}{l}\text { Control } \\
(n=45)\end{array}$ & $\mathbf{F}$ & Sig. & $\varepsilon^{2}$ & $\begin{array}{l}\text { Post- } \\
\text { hoc }^{5}\end{array}$ \\
\hline Edad & $73,37 \pm 8,78$ & $71,59 \pm 8,99$ & $73,76 \pm 7,45$ & $0,52(2 ; 122)$ & 0,60 & $-0,01$ & \\
\hline Años de educación & $10,80 \pm 5,03$ & $11,14 \pm 5,10$ & $13,11 \pm 4,60$ & $2,98(2 ; 118)$ & 0,51 & 0,03 & \\
\hline $\begin{array}{ll}\text { Sexo }^{\ddagger} & \\
\% \text { Mujeres } & (n) \\
\% \text { Hombres } & \text { (n) }\end{array}$ & $\begin{array}{ll}51,7 \% & (31) \\
48,3 \% & (29)\end{array}$ & $\begin{array}{ll}45,5 \% & (10) \\
54,5 \% & (12)\end{array}$ & $\begin{array}{ll}55,6 \% & (25) \\
44,4 \% & (20)\end{array}$ & $\chi^{2}=0,61(2)$ & 0,74 & & \\
\hline ACE-R-Ch & $50,80 \pm 18,62$ & $77,05 \pm 10,47$ & $89,13 \pm 9,13$ & $92,80(2 ; 124)$ & $<0,01^{*}$ & 0,59 & a \\
\hline MMSE & $17,80 \pm 5,92$ & $26,23 \pm 2,54$ & $27,84 \pm 2,27$ & $74,10(2 ; 124)$ & $<0,01$ * & 0,54 & a \\
\hline CDR & $1,90 \pm 0,83$ & $0,50 \pm 0,00$ & $0,00 \pm 0,00$ & $152,04(2 ; 124)$ & $<0,01 *$ & 0,71 & a \\
\hline ADLQ-Ch & $49,05 \pm 21,4$ & $20,12 \pm 11,05$ & $12,84 \pm 11,41$ & $63,31(2 ; 121)$ & $<0,01^{*}$ & 0,50 & b \\
\hline PFAQ & $15,92 \pm 9,54$ & $2,44 \pm 3,85$ & $0,64 \pm 1,51$ & $69,50(2 ; 119)$ & $<0,01^{*}$ & 0,53 & c \\
\hline IADL & $3,60 \pm 2,02$ & $6,21 \pm 2,12$ & $7,3 \pm 1,27$ & $51,19(2 ; 111)$ & $<0,01$ * & 0,47 & d \\
\hline AD8-Ch & $6,45 \pm 1,97$ & $3,31 \pm 1,70$ & $1,51 \pm 1,91$ & $78,33(2 ; 108)$ & $<0,01 *$ & 0,58 & b \\
\hline
\end{tabular}

Resultados Expresados en Media \pm Desviación Estándar. *La diferencia de medias es significativa. ${ }^{a}$ Demencia $<\mathrm{DCL}<\mathrm{Con}$ trol. ${ }^{\text {bDemencia }>D C L ~}>$ Control. ${ }^{\text {cDemencia }>D C L ; ~ D e m e n c i a ~}>$ Control. ${ }^{d}$ Demencia $<\mathrm{DCL}$; Demencia $<$ Control. ${ }^{*}$ Test Chi-Cuadrado, todas las demás comparaciones se realizaron mediante un análisis ANOVA de un factor. ${ }^{\circledR} E$ El análisis post-hoc se realizó con la prueba Games-Howell para todas las mediciones, excepto para el AD8-Ch en el que se ocupó la prueba Tukey.

Tabla 2. Puntajes de la versión chilena del ACE-R (ACE-R-Ch) divididos por dominio cognitivo

\begin{tabular}{|c|c|c|c|c|c|c|c|}
\hline \multirow[t]{2}{*}{ Parámetro } & \multicolumn{3}{|c|}{ Descriptivos según Grupos } & \multicolumn{4}{|c|}{ Comparaciones por Grupos } \\
\hline & $\begin{array}{l}\text { Demencia } \\
(n=60)\end{array}$ & $\begin{array}{c}\text { DCL } \\
(n=22)\end{array}$ & $\begin{array}{l}\text { Control } \\
(n=45)\end{array}$ & $\mathbf{F}$ & Sig. & $\varepsilon^{2}$ & $\begin{array}{l}\text { Post- } \\
\text { hoc }^{\S}\end{array}$ \\
\hline ACE-R-Ch & $50,80 \pm 18,62$ & $77,05 \pm 10,47$ & $89,13 \pm 9,13$ & $92,8 \quad(2 ; 124)$ & $<0,01 *$ & 0,59 & a \\
\hline Orientación y Atención & $10,25 \pm 4,49$ & $16,45 \pm 2,18$ & $17,29 \pm 1,34$ & $66,22(2 ; 124)$ & $<0,01 *$ & 0,51 & b \\
\hline Memoria & $8,88 \pm 5,47$ & $18,00 \pm 3,56$ & $22,62 \pm 3,83$ & $116,9(2 ; 124)$ & $<0,01 *$ & 0,65 & a \\
\hline Fluencias Verbales & $4,25 \pm 3,36$ & $7,32 \pm 2,57$ & $10,47 \pm 2,74$ & $54,44(2 ; 124)$ & $<0,01 *$ & 0,46 & a \\
\hline Lenguaje & $16,55 \pm 6,63$ & $22,18 \pm 3,20$ & $24,16 \pm 2,25$ & $32,51(2 ; 124)$ & $<0,01 *$ & 0,33 & a \\
\hline Habilidades Visoespaciales & $9,73 \pm 3,69$ & $13,23 \pm 2,05$ & $14,69 \pm 1,84$ & $39,63(2 ; 124)$ & $<0,01 *$ & 0,38 & a \\
\hline
\end{tabular}

Resultados Expresados en Media \pm Desviación Estándar. *La diferencia de medias es significativa. ${ }^{a}$ Demencia $<\mathrm{DCL}<\mathrm{Control}$.

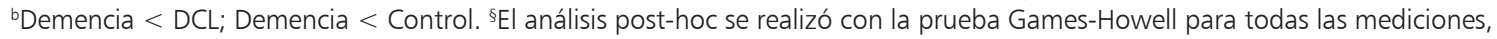
excepto para el dominio Fluencias Verbales en el que se ocupó la prueba Tukey.

de las evaluaciones de funcionalidad, IADL Scale y PFAQ, escalas en las que no se hallaron diferencias significativas $(\mathrm{p}>0,05)$ para los resultados entre el grupo de pacientes con DCL y los controles.

\section{$A C E-R-C h$}

En términos de variables demográficas, la prueba desarrollada no mostró diferencias significativas entre los resultados obtenidos según sexo $(\mathrm{t}=-1,508, \mathrm{p}>0,05)$, los que tampoco correlacio- naron significativamente con la edad $(r=-0,30$, $\mathrm{p}>0,05)$. Sí se encontró, sin embargo, una relación significativa entre los rendimientos en el ACE-RCh y los años de educación $(\mathrm{r}=0,475, \mathrm{p}<0,01)$.

En la Tabla 2 se sintetizan los puntajes del ACE-R-Ch para toda la muestra, detallando la división por dominio cognitivo que permite el instrumento y sus comparaciones pertinentes. Se encontraron así diferencias significativas $(\mathrm{p}<0,01)$ y de efecto moderado $\left(0,46<\varepsilon^{2}<0,65\right)$ entre los 
grupos diagnosticados en los puntajes totales y en los dominios Orientación y Atención, Memoria y Lenguaje, mientras que en los dominios Fluencias Verbales y Habilidades Visoespaciales, si bien se encontraron diferencias significativas $(\mathrm{p}<0,01)$, estas tuvieron un efecto menor $\left(0,33<\varepsilon^{2}<0,38\right)^{33}$. El análisis post-hoc mostró que los puntajes totales del ACE-R-Ch, así como los de Orientación y Atención, Memoria, Fluencias Verbales, Lenguaje y Habilidades Visoespaciales fueron significativamente más bajos $(\mathrm{p}<0,05)$ en el grupo de pacientes con demencia que en el de pacientes con DCL y que en el control. Los pacientes con DCL además obtuvieron rendimientos significativamente más bajos $(\mathrm{p}<0,05)$ que los controles en los puntajes totales, de Memoria, Fluencias Verbales, Lenguaje y Habilidades Visoespaciales, sin embargo, entre ambos grupos no se hallaron diferencias significativas en el dominio Orientación y Atención ( $p>0,05)$. Es posible señalar que todas las diferencias observadas en este último análisis sugieren ser de amplio rango (ver Anexo 2).

Tabla 3. Validez convergente de la versión chilena del ACE-R (ACE-R-Ch)

\begin{tabular}{|llc|}
\hline Evaluación & Instrumentos & $\begin{array}{c}\text { ACE-R- } \\
\text { Ch }\end{array}$ \\
\hline Eficiencia cognitiva global & MMSE & $0,952^{\ddagger}$ \\
Gravedad de la demencia & CDR & $-0,822^{\ddagger \ddagger}$ \\
\hline Funcionalidad en las acti- & ADLQ-Ch & $-0,70^{\ddagger}$ \\
vidades de la vida diaria & PFAQ & $-0,725^{\ddagger}$ \\
& IADL & $0,650^{\ddagger}$ \\
Cambios cognitivos & AD8-Ch & $-0,633^{\ddagger}$ \\
\hline
\end{tabular}

${ }^{\ddagger}$ Coeficiente de Correlación de Pearson, $p<0,001$. ${ }^{\ddagger}$ Coeficiente de Correlación rho de Spearman, $p<0,001$.

\section{Validez y Confiabilidad}

En términos de validez convergente, el ACE-RCh mostró correlaciones significativas con mediciones de eficiencia cognitiva global, gravedad de la demencia, capacidad funcional y cambios cognitivos $(\mathrm{p}<0,01)$ (Tabla 3). En cuanto a la confiabilidad, se estimó la consistencia interna del instrumento obteniéndose un coeficiente alfa de Cronbach de 0,918 , el que puede ser considerado como excelente.

\section{Utilidad Diagnóstica}

Las ABC obtenidas mediante las curvas ROC ejecutadas entregan valores más altos para el ACE-R-Ch que para el MMSE (Figura 1 y Tabla 4). Se consideró que el punto de corte que reveló el mejor ajuste entre sensibilidad y especificidad para el ACE-R-Ch fue el que contrastó el grupo de pacientes con demencia con los controles (punto de corte $=76$, sensibilidad $=0,917$, especificidad $=0,933$; Figura 1, i., Tabla 4, i.).

\section{Discusión}

Los resultados obtenidos permiten sostener que el ACE-R - Versión Chilena (ACE-R-Ch) es un instrumento válido, confiable y de buena utilidad diagnóstica para la detección de casos de demencia en una muestra de ancianos chilenos, lo que sugiere que su uso en la evaluación de quejas cognitivas a lo largo de Chile podría ser recomendable.

En la publicación original del ACE- ${ }^{9}$, Mioshi y colaboradores recomiendan dos puntos de corte, 82 puntos: sensibilidad $=0,84$, especificidad $=1,0$ y 88 puntos: sensibilidad $=0,94$, especificidad $=0,89$. El presente estudio propone que el punto de corte óptimo es el hallado para distinguir pacientes con demencia de sujetos sanos: 76 pun-

Tabla 4. Análisis con curvas ROC para el ACE-R-Ch y el MMSE

\begin{tabular}{|llccccc|}
\hline Comparación & $\begin{array}{l}\text { Instru- } \\
\text { mentos }\end{array}$ & $\begin{array}{c}\text { Área bajo } \\
\text { la curva }\end{array}$ & $\begin{array}{c}\text { Punto } \\
\text { de corte }\end{array}$ & Sensibilidad & $\begin{array}{c}\text { Especificidad } \\
\text { (Intervalo de } \\
\text { confianza al 95\%) }\end{array}$ \\
\hline i) Demencia vs control & ACE-R-Ch & 0,970 & 76 & 0,917 & 0,933 & 0,$942 ; 0,997$ \\
ii) Demencia y DCL vs control & MMSE & 0,955 & 25 & 0,883 & 0,889 & 0,$920 ; 0,990$ \\
& MMSE-R-Ch & 0,931 & 81 & 0,841 & 0,822 & 0,$889 ; 0,974$ \\
iii) DCL vs control & ACE-R-Ch & 0,827 & 91 & 0,864 & 0,756 & 0,$835 ; 0,944$ \\
& MMSE & 0,711 & 29 & 0,864 & 0,429 & 0,$730 ; 0,924$ \\
& & & 27 & 0,805 & 0,$586 ; 0,837$ \\
\hline
\end{tabular}




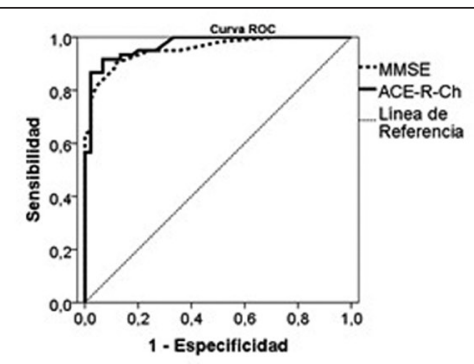

i) Demencia v/s Control

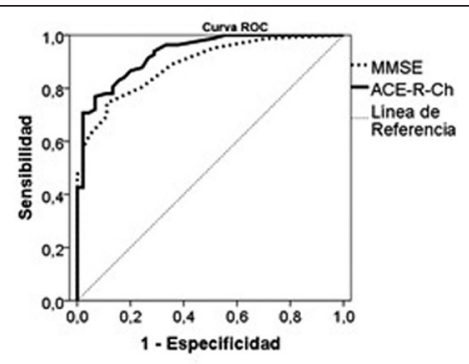

ii) Demencia y DCL v/s Control

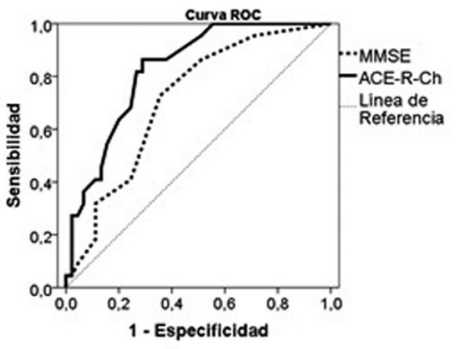

iii) DCL v/s Control

Figura 1. Curvas ROC para el ACE-R-Ch y el MMSE. ACE-R-Ch = Addenbrooke's Cognitive Examination - Revised - Versión Chilena; MMSE = Mini Mental State Examination.

tos, sensibilidad $=0,917$, especificidad $=0,933$, indicadores prácticamente idénticos a los arrojados por el estudio preliminar de esta investigación ${ }^{34}$. El punto de corte calculado para diferenciar pacientes con demencia y DCL, como grupo combinado, de controles también podría ser usado por presentar valores respetables: punto de corte $=81$, sensibilidad $=0,841$, especificidad $=0,822$, sin embargo, el punto de corte obtenido para diferenciar pacientes sólo con DCL de controles no se recomienda debido su baja especificidad: punto de corte $=91$, sensibilidad $=0,864$, especificidad $=0,622$.

En cuanto a validez convergente y confiabilidad, el ACE-R-Ch mostró resultados similares a los obtenidos en la publicación original ${ }^{9}$ que reportó validez convergente (correlación significativa $\mathrm{r}=-0,321, \mathrm{p}<0,001$ con la CDR) y confiabilidad (coeficiente alpha de Cronbach de 0,8) excelentes. Además, sobre este punto mostró resultados homólogos con las dos validaciones latinoamericanas conocidas hasta el momento: la brasileña ${ }^{35}$ y la argentina ${ }^{16}$. En pacientes con demencia, la utilidad diagnóstica, sensibilidad y especificidad del ACER-Ch también fueron parecidas a las encontradas por dichas investigaciones, aunque los puntos de corte fueron diferentes. En concreto, el estimado en Chile para distinguir entre pacientes con demencia y sujetos sanos fue más bajo que los hallados en los estudios mencionados, lo que podría explicarse por factores socioculturales ${ }^{10,36-38}$. Por otra parte, comparado con el MMSE, el ACE-R-Ch presentó mayor $\mathrm{ABC}$, lo que podría sugerir que es una prueba de mejor utilidad diagnóstica que el MMSE.

Una de las debilidades de esta investigación podría encontrarse en la inclusión exclusiva de sujetos de una unidad especializada en demencias dentro de un hospital. Habría sido valioso, por ejemplo, sumar a participantes de otros lugares especializados o centros de salud de atención primaria. Otro aspecto cuestionable de nuestro estudio podría vincularse al trabajo con pacientes diagnosticados con DCL si se tienen en cuenta las controversias que ha generado la definición de este concepto aún en construcción ${ }^{39}$.

En relación a la proyección de esta investigación, interesante resultaría trabajar en una normalización del ACE-R-Ch usando una muestra representativa de las personas de tercera edad en Chile.

En la actualidad es ampliamente conocido que múltiples desórdenes neurológicos (ACV, Parkinson, epilepsias, TEC, etc.) y psiquiátricos (esquizofrenia, TAB, etc.) cursan con trastornos cognitivos. En la práctica general o ambulatoria, ante quejas de problemas de memoria es necesario el uso de un test de screening breve y sensible que permita definir si hay motivos de preocupación que ameriten un estudio profundo ${ }^{40}$. Además de contar con adecuadas propiedades psicométricas, el test de screening efectivo debe ser de bajo costo y breve, junto con ser aceptado por la población objetivo ${ }^{2,41}$. En este trabajo se plantea que el ACE-R es un test que cumple con las características recién descritas, lo que lo deja bien posicionado para los ámbitos clínico e investigativo y se ve reflejado además en el interés que ha despertado en más de 150 centros, clínicos y de investigación, a nivel global (Portugal, Bélgica, Rumania, Australia, Nueva Zelanda, Sudáfrica, India, Sri Lanka, Argentina, Brasil, etc. $)^{9}$.

Para finalizar, si bien el ACE-R-Ch podría situarse como un instrumento que entrega elementos clínicos que aportan al diagnóstico de las demencias, es de resaltar que una evaluación neuropsicológica completa debería siempre incluir el testimonio de una fuente de garantía cercana 
al paciente sobre el estado de salud de este, la capacidad funcional en las AVD, la sintomatología neuropsiquiátrica respectiva, si es que la presenta, e informaciones respecto de posibles cambios cognitivos, a fin de obtener una visión íntegra del caso. De este modo, la utilización conjunta de pruebas de screening cognitivo y valoraciones de informantes en las demencias permitiría realizar un proceso de evaluación de mejor calidad ${ }^{42}$.
Agradecimientos: Nuestros más sinceros agradecimientos a la Dra. Eneida Mioshi, creadora de la versión original del ACE-R, a la Lic. Teresa Torralva, autora del artículo de la versión en español del ACE-R y además a sus respectivos equipos de trabajo. Agradecemos también a los Profesores del Departamento de Psicología de la Universidad de Chile Andrés Antivilo, Iris Gallardo y Elisabeth Wenk por su valiosa asesoría.

Anexo 2. Detalle análisis post-hoc de las valoraciones utilizadas

\begin{tabular}{|c|c|c|c|c|c|c|}
\hline \multirow[t]{3}{*}{ Parámetro } & \multicolumn{6}{|c|}{ Comparaciones Post-hoc } \\
\hline & \multicolumn{2}{|c|}{ Demencia vs Control } & \multicolumn{2}{|c|}{ Demencia vs DCL } & \multicolumn{2}{|c|}{ DCL vs Control } \\
\hline & Sig. & IC95\% & Sig. & IC95\% & Sig. & IC95\% \\
\hline ACE-R-Ch & $<0,01^{*}$ & $-44,91 ;-31,75$ & $<0,01^{*}$ & $-34,11 ;-18,38$ & $<0,01^{*}$ & $-18,47 ;-5,71$ \\
\hline Orientación y Atención ${ }^{a}$ & $<0,01^{*}$ & $-8,5 ; \quad-5,57$ & $<0,01^{*}$ & $-7,98 ; \quad-4,43$ & 0,24 & $-2,08 ; 0,41$ \\
\hline Memoria $^{a}$ & $<0,01^{*}$ & $-15,9 ;-11,58$ & $<0,01^{*}$ & $-11,61 ;-6,62$ & $<0,01^{*}$ & $-6,92 ;-2,32$ \\
\hline Fluencias Verbales $^{b}$ & $<0,01^{*}$ & $-7,63 ; \quad-4,8$ & $<0,01^{*}$ & $-4,86 ; \quad-1,28$ & $<0,01^{*}$ & $-5,02 ;-1,28$ \\
\hline Lenguaje $^{a}$ & $<0,01 *$ & $-9,8 ; \quad-5,41$ & $<0,01 *$ & $-8,25 ;-3,01$ & $0,04^{*}$ & $-3,84 ;-0,1$ \\
\hline Habilidades Visoespaciales $^{a}$ & $<0,01^{*}$ & $-6,27 ; \quad-3,64$ & $<0,01^{*}$ & $-5,04 ; \quad-1,95$ & $0,02^{*}$ & $-2,72 ;-0,2$ \\
\hline MMSE $^{a}$ & $<0,01^{*}$ & $-12,04 ;-8,05$ & $<0,01^{*}$ & $-10,67 ; \quad-6,19$ & $0,04^{*}$ & $-3,18 ;-0,06$ \\
\hline $\mathrm{CDR}^{\mathrm{a}}$ & $<0,01^{*}$ & 1,$64 ; \quad 2,16$ & $<0,01^{*}$ & 1,$14 ; \quad 1,66$ & $<0,01^{*}$ & 0,$5 ; \quad 0,5$ \\
\hline ADLQ-Ch ${ }^{a}$ & $<0,01^{*}$ & 28,$41 ; 44,01$ & $<0,01^{*}$ & 20,$09 ; 37,75$ & $0,047^{*}$ & 0,$08 ; 14,5$ \\
\hline $\mathrm{PFAQ}^{\mathrm{a}}$ & $<0,01^{*}$ & 12,$27 ; 18,29$ & $<0,01^{*}$ & 9,$81 ; 17,14$ & 0,16 & $-0,57 ; 4,19$ \\
\hline $\mathrm{ADL}^{\mathrm{a}}$ & $<0,01^{*}$ & $-4,5 ; \quad-2,9$ & $<0,01^{*}$ & $-3,99 ;-1,24$ & 0,12 & $-2,4 ; \quad 0,22$ \\
\hline AD8-Ch ${ }^{b}$ & $<0,01^{*}$ & 3,$96 ; \quad 5,85$ & $<0,01^{*}$ & 1,$85 ; \quad 4,43$ & $<0,01^{*}$ & 0,$42 ; 3,11$ \\
\hline
\end{tabular}

$\mathrm{IC95 \%}=$ Intervalo de Confianza al 95\%. * La diferencia de medias es significativa. ${ }^{\mathrm{a} T e s t}$ de Levene $p<0,05$. Post-hoc Games-

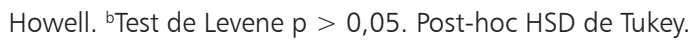

\section{Referencias}

1. Folstein MF, Folstein SE, Mchugh PR. "Mini-mental state". A practical method for grading the cognitive state of patients for the clinician. J Psychiatr Res 1975; 12: 189-98.

2. González-Hernández J, Aguilar L, Oporto S, Araneda L, Vásquez M, Von Bernhardi R. Normalización del "MiniMental State Examination” según edad y educación, para la población de Santiago de Chile. Revista Memoriza. com 2009; 3: 23-34.

3. Mathuranath PS, Nestor PJ, Berrios GE, Rakowicz W, Hodges JR. A brief cognitive test battery to differentiate Alzheimer's disease and frontotemporal dementia. Neurology 2000; 55: 1613-20.

4. Bak TH, Rogers TT, Crawford LM, Hearn VC, Mathuranath PS, Hodges JR. Cognitive bedside assessment in atypical parkinsonian syndromes. J Neurol Neurosurg Psychiatry 2005; 76: 420-2.

5. Bier JC, Ventura M, Donckels V, Eyll E, Claes T, Slama $\mathrm{H}$, et al. Is the Addenbrooke's cognitive examination effective to detect frontotemporal dementia? J Neurol 2004; 251: 428-31.

6. Galton CJ, Erzinçlioglu S, Sahakian BJ, Antoun N, Hodges JR. A comparison of the Addenbrooke's Cognitive Examination (ACE), conventional neuropsychological assessment, and simple MRI-based medial temporal lobe evaluation in the early diagnosis of Alzheimer's disease. Cogn Behav Neurol 2005; 18: 144-50.

7. Larner AJ. An audit of the Addenbrooke's Cognitive Examination (ACE) in clinical practice. Int J Geriatr Psychiatry 2005; 20: 593-4.

8. Dudas RB, Berrios GE, Hodges JR. The Addenbrooke's cognitive examination (ACE) in the differential diagnosis of early dementias versus affective disorder. Am J Geriatr Psychiatry 2005; 13: 218-26.

9. Mioshi E, Dawson K, Mitchell J, Arnold R, Hodges JR. The Addenbrooke's Cognitive Examination Revised (ACE-R): A brief cognitive test battery for dementia screening. Int J Geriatr Psychiatry 2006; 21: 1078-85.

10. Peña-Casanova J, Blesa R, Aguilar M, GramuntFombuena N, Gómez-Ansón B, Oliva R, et al. Spanish Multicenter Normative Studies (NEURONORMA Project): Methods and sample characteristics. Arch Clin Neuropsychol 2009; 24: 307-19.

11. Espino DV, Lichtenstein MJ, Palmer RF, Hazuda HP. Evaluation of the mini-mental state examination's internal consistency in a community-based sample of MexicanAmerican and European-American elders: Results from the San Antonio Longitudinal Study of Aging. J Am Geriatr Soc. 2004; 52: 822-7. 
12. Lawrence J, Davidoff D, Katt-Lloyd D, Auerbach M, Hennen J. A pilot program of improved methods for community-based screening for dementia. Am J Geriatr Psychiatry 2001; 9: 205-11.

13. Lorentz WJ, Scanlan JM, Borson S. Brief screening tests for dementia. Can J Psychiatry 2002; 47: 723-33.

14. Roca M, Manes F. Test de screening para demencias. En: Labos E, Slachevsky A, Fuentes P, Manes F, editores. Tratado de Neuropsicología Clínica: bases conceptuales y técnicas de evaluación. (1 ${ }^{\text {a }}$ ed.). Buenos Aires, Argentina: Akadia; 2008. p. 473-81.

15. Slachevsky A, Villalpando JM, Sarazin M, Hahn-Barma V, Pillon B, Dubois B. Frontal assessment battery and differential diagnosis of frontotemporal dementia and Alzheimer disease. Arch Neurol 2004; 61: 1104-7.

16. Torralva T, Roca M, Gleichgerrcht E, Bonifacio A, Raimondi C, Manes F. Validación de la versión en español del Addenbrooke's Cognitive Examination-Revised (ACE-R). Neurología 2011; 26: 351-6.

17. Hughes CP, Berg L, Danziger WL, Coben LA, Martin RL. A new clinical scale for the staging of dementia. $\mathrm{Br} J$ Psychiatry 1982; 140: 566-72.

18. Morris JC. The Clinical Dementia Rating (CDR): Current version and scoring rules. Neurology 1993; 43: 2412-4.

19. Yesavage JA. Geriatric Depression Scale. Psychopharmacol Bull 1988; 24: 709-11.

20. Zung WWK. A rating instrument for anxiety disorders. Psychosomatics 1971; 12: 371-9.

21. Mckhann G, Drachman D, Folstein M, Katzman R, Price D, Stadlan EM. Clinical diagnosis of Alzheimer's disease: Report of the NINCDS-ADRDA Work Group under the auspices of Department of Health and Human Services Task Force on Alzheimer's Disease. Neurology 1984; 34: 939-44.

22. Neary D, Snowden JS, Gustafson L, Passant U, Stuss D, Black S, et al. Frontotemporal lobar degeneration: A consensus on clinical diagnostic criteria. Neurology 1998; 51: 1546-54.

23. Roman GC, Tatemichi TK, Erkinjuntti T, Cummings JL, Masdeu JC, García JH, et al. Vascular dementia: Diagnostic criteria for research studies. Report of the NINDS-AIREN International Workshop. Neurology 1993; 43: 250-60.

24. Mckeith IG, Galasko D, Kosaka K, Perry EK, Dickson DW, Hansen LA, et al. Consensus guidelines for the clinical and pathologic diagnosis of dementia with Lewy bodies (DLB): Report of the consortium on DLB international workshop. Neurology 1996; 47: 1113-24.

25. Petersen RC. Mild cognitive impairment: transition between aging and Alzheimer's disease. Neurología 2000; 15: 93-101.

26. Muñoz C, Riveros R, Flores P, Wenk E, Slachevsky A. Activities of Daily Living Questionnaire ADLQ -Versión Chilena-: propiedades psicométricas y utilidad diagnóstica incluyendo una sub-escala de tecnología. Rev Chil
Neuro-Psiquiatr 2010; 48 (Supl. 2): 117.

27. Quiroga P, Albala C, Klaasen G. Validación de un test de tamizaje para el diagnóstico de demencia asociada a la edad, en Chile. Rev Med Chile 2004; 132: 467-78.

28. Lawton MP, Brody EM. Assessment of older people: Selfmaintaining and instrumental activities of daily living. Gerontologist 1969; 9: 179-86.

29. Muñoz C, Núñez J, Flores P, Behrens MI, Slachevsky A. Utilidad de un cuestionario breve dirigido al informante para el diagnóstico temprano de casos de demencia: la versión chilena del AD8 (AD8-Ch). Rev Med Chile 2010; 138: 1063-5.

30. Gifford DR, Cummings JL. Evaluating dementia screening tests: Methodologic standards to rate their performance. Neurology 1999; 52: 224-7.

31. Bland JM, Altman DG. Cronbach's alpha. BMJ 1997; 314: 572.

32. Bewick V, Cheek L, Ball J. Statistics review 13: Receiver operating characteristic curves. Crit Care 2004; 8: 508-12.

33. Cohen J. Statistical power analysis for the behavioral sciences. ( $2^{\mathrm{a}}$ ed.). Hillsdale, NJ: Lawrence Erlbaum Associates; 1988.

34. Muñoz C, Henríquez F, Sánchez M, Flores P, Slachevsky A. Validación del Addenbrooke's Cognitive Examination-Revised (ACE-R) en Chile: datos preliminares. Rev Chil Neuro-Psiquiatr 2010; 48 (Supl. 2): 117.

35. Carvalho VA, Barbosa MT, Caramelli P. Brazilian version of the Addenbrooke Cognitive Examination-Revised in the diagnosis of mild Alzheimer disease. Cogn Behav Neurol 2010; 23: 8-13.

36. Ostrosky-Solís F, Ardila A, Rosselli M, López-Arango G, Uriel-Mendoza V. Neuropsychological test performance in illiterate subjects. Arch Clin Neuropsychol 1998; 13: 645-60.

37. Ostrosky-Solís F, Canseco E, Quintanar L, Navarro E, Meneses S, Ardila A. Sociocultural effects in neuropsychological assessment. Int J Neurosci 1985; 27: 53-66.

38. Finlayson MA, Johnson KA, Reitan RM. Relationship of level of education to neuropsychological measures in brain-damaged and non-brain-damaged adults. J Consult Clin Psychol 1977; 45: 536-42.

39. Chertkow H, Nasreddine Z, Joanette Y, Drolet V, Kirk J, Massoud F, et al. Mild cognitive impairment and cognitive impairment, no dementia: Part $\mathrm{A}$, concept and diagnosis. Alzheimers Dement 2007; 3: 266-82.

40. Hatfield CF, Dudas RB, Dening T. Diagnostic tools for dementia. Maturitas 2009; 63: 181-5.

41. Berg L, Mckeel Jr. DW, Miller JP, Storandt M, Rubin EH, Morris JC, et al. Clinicopathologic studies in cognitively healthy aging and Alzheimer's disease: Relation of histologic markers to dementia severity, age, sex, and apolipoprotein E genotype. Arch Neurol 1998; 55: 326-35.

42. Jorm AF. The Informant Questionnaire on Cognitive Decline in the Elderly (IQCODE): A review. Int Psychogeriatr 2004; 16: 275-93. 